\title{
Harmful competition in insurance markets
}

\author{
Giuseppe DE FEO ${ }^{1}$ and Jean HINDRIKS ${ }^{2}$
}

June 13, 2014

\begin{abstract}
There is a general presumption that competition is a good thing. In this paper we show that competition in the insurance markets can be bad and that adverse selection is in general worse under competition than under monopoly. The reason is that monopoly can exploit its market power to relax incentive constraints by cross-subsidization between different risk types. Cream-skimming behavior, on the contrary, prevents competitive firms from using implicit transfers. In effect monopoly is shown to provide better coverage to those buying insurance but at the cost of limiting participation to insurance. Performing simulation for different distributions of risk, we find that monopoly in general performs (much) better than competition in terms of the realization of the gains from trade across all traders in equilibrium.
\end{abstract}

Keywords: monopoly, competition, insurance, adverse selection.

JEL Classification: D41, D42, D82, G22

\footnotetext{
${ }^{1}$ Department of Economics, University of Strathclyde, Glasgow, UK and Dipartimento di Scienze Economiche e Aziendali, Università di Pavia. E-mail: giuseppe.defeo@strath.ac.uk

${ }^{2}$ CORE, Université catholique de Louvain, Belgium. E-mail: jean.hindriks@uclouvain.be

We are grateful to Enrico Minelli, Francesca Stroffolini, Piero Tedeschi, Izabela Jelovac, Pierre Pestieau, François Maniquet, Tito Pietra, Paolo Siconolfi, Alberto Bennardo, Marcello D'Amato, John Moore, Gaetano Bloise, Mario Tirelli, John Hey, Peter Spencer and Tore Nilssen for helpful comments and suggestions. This work also benefitted from comments from seminar audiences at CORE, Porto Conte, London School of Economics and the Universities of Milano Bocconi, Liege, Leuven, Salerno, Napoli, Edinburgh, Roma TRE, Ljubljana, and York. The scientific responsibility is assumed by the authors.
} 


\section{Introduction}

In this paper we address the critical question: how and how well competition on the markets handles the fundamental problems of information. With imperfect information, market actions or choices convey information and we know from earlier work (e.g. Rothschild and Stiglitz, 1976) that inefficiency and even existence problems can arise in competitive markets because the slight change in the action of the informed side of the market discretely changes beliefs of the other side of the market. While information asymmetries inevitably arise, the extent to which they do so and their consequences on the realization of the gains from trade depend on how the market is structured. This raises the fundamental question of the interplay between two forms of market imperfections: imperfect information and imperfect competition. There is no particular reason why competition should be better in the presence of imperfect information. The simplest way by which this would not be true is when the firm could exploit its market power to relax the incentive constraints.

The aim of this paper is to evaluate the efficiency of competition on the insurance market in the presence of adverse selection. Using the benchmark model of Rothschild and Stiglitz (1976), we contrast the competitive equilibrium outcome with the monopoly equilibrium outcome à la Stiglitz (1977) and we compare their relative efficiency. Following Rustichini et al. (1994), the (expected) efficiency of an equilibrium is the fraction whose numerator is the expected gains from trade across all traders in the equilibrium and whose denominator is the gains from trade across all traders with full information. Using this criterion we compare the monopoly outcome with one seller of insurance contracts and many potential buyers with different risks against the competitive outcome imposing zero profit on each contract that might be offered in equilibrium. With a continuum of types it is well known that a competitive equilibrium à la Rothschild-Stiglitz does not exist. To address this issue, some alternative equilibrium concepts, based either on game theoretic refinements or on a Walrasian approach, have been proposed in order to ensure the existence of competitive equilibria. Even though no general agreement has been reached so far about the equilibrium concept, the general intuition in the classical as well as more recent literature is that competition typically results in the provision of a set of contracts that fully separate types (Chiappori, 2006). In this paper we refer to the concept of reactive equilibrium developed by Riley (1979) and Engers and Fernandez (1987) for which the Pareto-dominant full separating zero-profit outcome is the unique reactive equilibrium. ${ }^{1}$

The key finding is that the monopoly outcome, in general, is more efficient than the competitive outcome (according to our expected efficiency criterion). The reason why monopoly performs better than competition is that the monopolist can exploit its market power to offer contracts that better satisfy the incentive constraints. More precisely, the monopolist can offer contracts with implicit transfers across agent types that can relax the incentive constraints and

\footnotetext{
${ }^{1}$ This is also the equilibrium allocation achieved in a Walrasian equilibrium (Bisin and Gottardi, 2006). See also Hellwig (1987) and Dubey and Geanakoplos (2002) for other equilibrium concepts leading to the same resource allocation.
} 
implement a larger set of allocations. This is one of many examples of the interplay between market imperfections (see Jaffe and Stiglitz, 1990; Stiglitz, 1975). The economy, in effect has to trade off between two different imperfections: imperfections of information or imperfections of competition, with no particular reason that these imperfections will be balanced optimally. As we shall see, competition provides all risk types with an insurance contract, but coverage is only partial. On the contrary, under monopoly low risk types are forced to quit the market, but coverage increases for the participating risk types.

Our paper continues a line of research begun by Stiglitz (1977), who analyzed monopoly insurance markets, and compared the equilibrium outcome with the (two-type) competitive outcome. In his qualitative comparison Stiglitz shows how the high-risk consumers always buy full coverage at a price larger than the fair premium, while the low risks buy partial insurance, both under monopoly and competition. A further qualitative comparison is performed by Dahlby (1987) in the same two-type framework and shows that monopoly may be better than competition when there are enough low types. In our analysis with a continuum of types we show that monopoly performs better than competition for almost any possible distribution of risk types, and not only in the case of a large proportion of low-risk types; i.e., when insurance generates little surplus. ${ }^{2}$

We perform this analysis in a non-expected utility framework using the dual theory approach to choice under risk developed by Yaari (1987). It turns out that by using this specification of individual preferences we are able to provide a clear-cut comparison between monopoly and competition. The dual theory has the property that utility is linear in income, and risk aversion is expressed entirely by a transformation of probabilities in which bad outcomes are given relatively higher weights and good outcomes are given relatively lower weights. In our simple two-state model the probability of bad outcome is weighted up by a loading factor. It would be absurd to suggest that the dual theory provides a better model than the expected utility. The latter has obvious appeal and has provided so many useful results in insurance theory. Nonetheless, we feel there is some gain from studying the properties of our simple non-expected utility model, even if only to derive some clear insights on the efficiency of competition in the presence of adverse selection. ${ }^{3}$

A similar approach is used by Attar et al. (2011) who analyze a model of adverse selection à la à la Akerlof (1970) but with divisible goods. As in our model, they consider agents with linear preferences both on quantities (which is coverage in insurance markets) and money.

\footnotetext{
${ }^{2}$ In a different setting, a labour market where workers suffer from social comparison, von Siemens (2012) analyzes the competitive equilibrium when workers' ability is private information. He shows that competition between firms generate rent extraction by skilled workers, which in turn increases inequality and may provoke inefficient sorting into unemployment. If there is only one firm in the economy, this monopsonist can reduce rent extraction by skilled workers and the differences in the wage workers receive. Interestingly, it is the firm's ability to perform cross-subsidization that increases the efficiency of the monopsonist equilibrium.

${ }^{3}$ Another distinctive property of insurance under dual theory is that the demand of insurance cannot decrease with wealth. In contrast, the expected utility model makes the comparison between competition and monopoly difficult since by charging a higher premium (relative to competition) for a given coverage the monopoly increases the marginal willingness to pay for insurance.
} 
However, they focus on nonexclusive contracts and show that, contrary to our result, the competitive equilibrium entails extreme choices for the agents depending on their type: either the whole endowment is sold or no treed occurs (in the insurance setting this equilibrium would be that some types get full insurance and all the other types do not buy any coverage). In the present paper, where we focus on exclusive contracts, we show that the competitive equilibrium is characterized by a continuum of partial coverage contract monotonically increasing with the risk type of the agent and only the highest risk type gets full coverage. It is only under monopoly that we have a result qualitatively similar to Attar et al. (2011): a pool of high risk types buys a full coverage contract, while all the other types buy no contract at all.

A line of research that is closely related to non-expected utility theory considers the distortion in the probabilities as a result of biased beliefs of the agents. In this framework, Sandroni and Squintani $(2007,2012)$ analyze the monopoly and competitive equilibria in an insurance market where overconfidence of (a subset of) consumers alters the behavior of otherwise standard expected utility maximizers in a market à la Rotschild Stiglitz (1976). They show that contrary to the standard result, the competitive equilibrium does depend on the composition of (perceived and actual) risk in the population, while the presence of overconfident agents is equivalent to a change of the risk composition for the monopoly. They also show that the competition as well as monopoly can lead to no insurance to low risks individuals, in their two-type framework. In a more general framework which takes into account both moral hazard and adverse selection problems de Meza and Webb (2001) Jullien et al. (2007) De Donder and Hindriks (2009) analyze (competitive and monopolistic) screening when more risk averse individuals exert more preventive care and have also higher willingness to pay for issuance. In such a case, and contrary to the standard results in Rothschild and Stiglitz (1976), a negative correlation may (but non necessarily will) arise between risk and insurance. When biased beliefs are included in such framework, Spinnewijn (2013) provides conditions for positive and negative correlation between risk and coverage. Interestingly for our analysis, using the full information case as a benchmark, Spinnewijn (2013) shows that asymmetric information makes the competitive equilibrium inefficient while, on the contrary, some consumers may benefit from information asymmetry in a monopolistic market; as a result, heterogeneity in risk perception may strengthen the scope for public intervention even in competitive markets.

The paper is organized as follows. In Section 2 we present the model. Section 3 contains the monopolist and competitive equilibria with a continuum of types. In Section 4 simulation results are provided about efficiency of competitive and monopoly markets. Section 5 concludes.

\section{The model}

There are two possible states of the world: the "no accident" state and the "accident" state. Individuals differ only by their probability of accident, in which case they face a (fixed) damage $d=1$. There is no moral hazard since individuals cannot affect their probability of accident which is fixed. There is a continuum of risk in the economy distributed according to 
a cumulative probability function $F(\theta)$ with density $f(\theta)>0$ on a closed and compact interval $\theta \in[\underline{\theta}, \bar{\theta}]$ (with $0 \leq \underline{\theta}<\bar{\theta}<1)$.

Adverse selection is introduced by assuming that individual risk is private information, while the distribution of risks is common knowledge. We model individuals' risk preferences using Yaari (1987)'s dual theory (DT). We first give a general description of this approach before applying it to our model. Let wealth $X$ be a random variable distributed over $[\underline{x}, \bar{x}]$ according to the distribution function $\Psi(x)$. Yaari's representation of preferences is dual to the expected utility theory $(\mathrm{EU})$ in the sense that it is linear in wealth but non linear in probabilities. Probabilities are transformed by a function $\Phi($.$) defined on the distribution function \Psi(x) .{ }^{4}$ More precisely, DT preferences over $X$ are given by

$$
V(X)=\int x \Phi^{\prime}(\Psi(x)) d \Psi(x)
$$

where $\Phi(0)=0, \Phi(1)=1$ and $\Phi^{\prime}()>.0 . \Phi^{\prime}($.$) are non-negative weights adding up to one.$ Attitude towards risk is conveyed entirely by the shape of $\Phi($.$) . Risk aversion is characterized$ by the concavity of $\Phi($.$) , i.e. \Phi^{\prime \prime}()<$.0 . In this case, bad outcomes (with low $\Psi(X)$ ) receive higher weights than good outcomes (with high $\Psi(X)$ ). In other words, $V(X)$ is the certaintyequivalent of $X$ computed as a weighted average of outcomes in which bad outcomes are given high weight while good outcomes are given low weight. Since $V(X)$ is linear in wealth, this approach separates attitude towards risk from attitude towards wealth.

We now apply DT to our simple two-state setting. For an individual with wealth $w$ facing a damage $d=1$ with probability $\theta$, an insurance contract $\{\delta, \pi\}$ with coverage rate $\delta \in[0,1]$ and premium $\pi>0$ yields the random variable $X=(w-\pi-(1-\delta), \theta ; w-\pi, 1-\theta)$. We thus define the utility associated to this insurance contract as

$$
\begin{aligned}
V(\theta ; \delta, \pi) & =\phi(\theta)(w-\pi-(1-\delta))+(1-\phi(\theta))(w-\pi) \\
& =w-\pi-\phi(\theta)(1-\delta)
\end{aligned}
$$

where risk aversion is represented by $\phi(\theta)>\theta$ (and $1-\phi(\theta)<1-\theta$ ). ${ }^{5}$ In this paper, we further assume that $\phi(\theta)=(1+\alpha) \theta$, with $0 \leq \alpha \leq \frac{1-\bar{\theta}}{\bar{\theta}}$ (the upper bound guaranteeing that $\phi(\theta) \leq 1 \forall \theta$ ). Making $\alpha$ independent of $w$ accords with our desire to disentangle risk aversion from income and will greatly simplify the analysis. Using this formulation, type- $\theta$ utility function from insurance contract $\{\delta, \pi\}$ is

$$
V(\theta ; \delta, \pi)=\omega-\pi-(1+\alpha)(1-\delta) \theta
$$

where the utility loss from the residual risk $(1-\delta) \theta$ is inflated by the markup factor $1+\alpha$.

\footnotetext{
${ }^{4}$ Alternatively this probability transformation function could be defined on the decumulative distribution function $1-\Psi$ such as in Yaari (1987).

${ }^{5}$ Note that in our model with a discrete random variable, risk aversion translates into the transformation of the discrete density function $\phi(\theta)>\theta$ rather than the concave transformation of the distribution function $\Phi^{\prime \prime}(\Psi)<0$ as for continuous random variable. In both cases risk aversion implies that bad outcomes are given higher weight and good outcomes lower weight.
} 
Now, comparing the utility with insurance against the utility without insurance we can define the reservation premium for each type. This is the premium $\tilde{\pi}(\theta)$ that solves

$$
\begin{aligned}
V(\theta ; \delta, \pi) & =V(\theta ; 0,0) \\
\omega-\pi-(1+\alpha)(1-\delta) \theta & =\omega-(1+\alpha) \theta
\end{aligned}
$$

so that the reservation premium of type $\theta$ for coverage $\delta$ is:

$$
\tilde{\pi}(\theta ; \delta)=(1+\alpha) \delta \theta
$$

Moreover the surplus of the agent is defined as the difference between the reservation price and the price effectively paid:

$$
\begin{aligned}
S(\theta ; \delta, \pi) & =\tilde{\pi}(\delta ; \theta)-\pi \\
& =(1+\alpha) \delta \theta-\pi
\end{aligned}
$$

Assuming $\pi>0$, with free participation, those agents for which $\tilde{\pi}(\theta ; \delta)<\pi$ will drop out of the market.

It is straightforward to see that the functions $V$ and $S$ have the Single-Crossing property in the contract space- $(\delta, \pi)$, because the marginal value of coverage is increasing in $\theta$.

\section{Monopoly versus competition equilibrium outcomes}

In this section we study the equilibrium outcomes of monopoly and competition in an insurance market with a continuum of risks.

The optimization problem of the monopolist is:

$$
\max _{\pi(\theta), \delta(\theta)} \int_{\underline{\theta}}^{\bar{\theta}}[\pi(\theta)-\delta(\theta) \theta] d F(\theta)
$$

subject to

$$
\begin{aligned}
& V(\theta ; \delta(\theta), \pi(\theta)) \geq V(\theta ; 0,0) \quad \forall \theta \in[\underline{\theta}, \bar{\theta}] \\
& V(\theta ; \delta(\theta), \pi(\theta)) \geq V(\theta ; \delta(\widehat{\theta}), \pi(\widehat{\theta})) \quad \forall \theta, \widehat{\theta} \in[\underline{\theta}, \bar{\theta}]
\end{aligned}
$$

where (2) is the set of participation constraints and (3) denotes the set of incentive constraints. Analyzing the set (2) we can see that

$$
V(\underline{\theta} ; \delta(\underline{\theta}), \pi(\underline{\theta})) \geq V(\underline{\theta} ; 0,0)
$$

must be binding, for otherwise it would be possible to increase $\pi(\theta) \forall \theta>\underline{\theta}$. This is the classical monopoly result of full rent extraction at the bottom.

In the following Proposition the monopolist outcome is summarized. 
Proposition 1 In a monopoly insurance market with a continuum of risks, there exists

$$
\theta^{*}=\frac{1+\alpha}{\alpha h\left(\theta^{*}\right)}
$$

with $h($.$) the non-decreasing hazard rate function, such that the equilibrium contracts are$

$$
\begin{array}{ll}
\left\{\delta^{m}(\theta), \pi^{m}(\theta)\right\}=\{0,0\} & \forall \theta \in\left[\underline{\theta}, \theta^{*}\right] \\
\left\{\delta^{m}(\theta), \pi^{m}(\theta)\right\}=\left\{1,(1+\alpha) \theta^{*}\right\} & \forall \theta \in\left[\theta^{*}, \bar{\theta}\right]
\end{array}
$$

Proof. See Appendix.

Therefore the solution is characterized by a (pooling) contract that offers full coverage to all $\theta \geq \theta^{*}$ with a premium extracting the entire surplus from type $\theta^{*}$ and no insurance to all $\theta<\theta^{*}$. The equilibrium payoff of type $\theta$ under monopoly is:

$$
\begin{array}{lll}
V\left(\theta ; \delta^{m}(\theta), \pi^{m}(\theta)\right)=\omega-(1+\alpha) \theta & \forall \theta \in\left[\underline{\theta}, \theta^{*}\right) \\
V\left(\theta ; \delta^{m}(\theta), \pi^{m}(\theta)\right)=\omega-(1+\alpha) \theta^{*} & \forall \theta \in\left[\theta^{*}, \bar{\theta}\right]
\end{array}
$$

Monopolist (per capita) profit is

$$
\Pi^{m}=(1+\alpha) \theta^{*}\left[1-F\left(\theta^{*}\right)\right]-\int_{\theta^{*}}^{\bar{\theta}} \theta d F(\theta)
$$

Rewriting $h\left(\theta^{*}\right)=\frac{f\left(\theta^{*}\right)}{1-F\left(\theta^{*}\right)}$ the pivotal type solves

$$
\alpha \theta^{*} f\left(\theta^{*}\right)=(1+\alpha)\left(1-F\left(\theta^{*}\right)\right)
$$

where the LHS is the revenue loss of an increase in $\theta^{*}$ due to the non-participation of pivotal type and the RHS is the revenue gain from charging a higher price on all agents above the pivotal type $\theta^{*}$.

The pooling contract performs cross-subsidization among types. In fact, while $\theta^{*}$-type individuals are extracted the whole surplus, higher types are left with some rent and may even pay a premium lower than the fair price.

Shifting to the analysis of competition, it is well known that with a continuum of types a competitive equilibrium may fail to exist. In fact Riley (2001) showed the general nonexistence of the Rothschild-Stiglitz equilibrium. This existence problem can be circumvented by resorting to the reactive equilibrium concept introduced by Riley (1979) and developed further by Engers and Fernandez (1987). A reactive equilibrium is a set of offers such that there is no profitable deviation by any firm given that other firms can optimally react to this deviation by offering new contracts. Engers and Fernandez (1987) provide general conditions, for which the Pareto-dominant full-separating zero-profit set of contracts is the unique reactive 
equilibrium outcome. ${ }^{6}$ It turns out that those conditions hold true in our framework. ${ }^{7}$ The key element is that firms are deterred to deviate from the full separating equilibrium by the belief that other firms will react to "skim the cream" and make such initial deviation unprofitable.

The Pareto-dominant fully separating zero-profit competitive equilibrium solves

$$
\max _{\substack{\pi(.) \geq 0 \\ \delta(.) \in[0,1]}} V(\theta ; \delta(\theta), \pi(\theta)) \quad \forall \theta=[\underline{\theta}, \bar{\theta}]
$$

subject to (2), (3) and the additional zero profit constraint:

$$
\pi(\theta)-\delta(\theta) \theta=0 \quad \forall \theta \in[\underline{\theta}, \bar{\theta}]
$$

The following Proposition summarizes the result due to Hindriks and De Donder (2003).

Proposition 2 In a competitive insurance market with a continuum of risks, the reactive equilibrium is characterized by the following set of contracts:

$$
\left\{\delta^{c}(\theta), \pi^{c}(\theta)\right\}=\left\{(\theta / \bar{\theta})^{\frac{1}{\alpha}}, \theta(\theta / \bar{\theta})^{\frac{1}{\alpha}}\right\} \quad \forall \theta \in[\underline{\theta}, \bar{\theta}]
$$

Proof. See Appendix.

The equilibrium payoff of type $\theta$ under competition is then:

$$
V\left(\theta ; \delta^{c}(\theta), \pi^{c}(\theta)\right)=\omega-(1+\alpha) \theta+\alpha \theta(\theta / \bar{\theta})^{\frac{1}{\alpha}} \quad \forall \theta \in[\underline{\theta}, \bar{\theta}]
$$

So, while in the monopoly every type $\theta \in\left[\theta^{*}, \bar{\theta}\right]$ gets full insurance, with competition only the highest-risk individuals obtain full coverage and all the other individuals with lower risk obtain partial coverage. On the other hand, every $\theta \in\left[\underline{\theta}, \theta^{*}\right)$ gets no insurance with monopoly, while they are provided at least with partial coverage in the competitive case. Figure 1 compares equilibrium coverage with monopoly and competition for a given distribution of risks.

The crucial feature of competitive equilibrium is that the set of implementable contracts is smaller than under monopoly. Since each contract must break even - by the constraint (7) - no cross-subsidization can be performed among types. As a consequence, the distribution of risks in the population does not influence the equilibrium outcome. In fact, there is a unique solution to the problem of maximizing the utility of each type given that every contract must break even and must be incentive compatible.

\footnotetext{
${ }^{6}$ Notice that this set of contracts is the Rothschild-Stiglitz candidate equilibrium. Many other equilibrium concepts have been put forward, such as those developed by Hellwig (1987), Dubey and Geanakoplos (2002), and the Walrasian approach developed by Prescott and Townsend (1984), but all implement the same allocation.

${ }^{7}$ The conditions for existence and uniqueness of a reactive equilibrium in our model are: (1) a continuous probability distribution $F(\theta)$; (2) the profit function of insurance firms is continuous, bounded and non increasing in $\theta$ and $\delta$; (3) $V(\theta ; \delta, \pi)$ is continuous on $\Theta \times \Delta \times \Pi$ where $\Delta=[0,1]$ and $\Pi=[\underline{\pi}, \bar{\pi}]$ with $\underline{\pi}=\inf \{\tilde{\pi}(\theta ; \delta): \theta \in \Theta, \delta \in \Delta\}$ and $\bar{\pi}=\sup \{\tilde{\pi}(\theta ; \delta): \theta \in \Theta, \delta \in \Delta\}$, is strictly decreasing in $\pi$ and satisfies the Single-Crossing property; (4) the contract space is a closed set $\Delta \times \Pi$.
} 


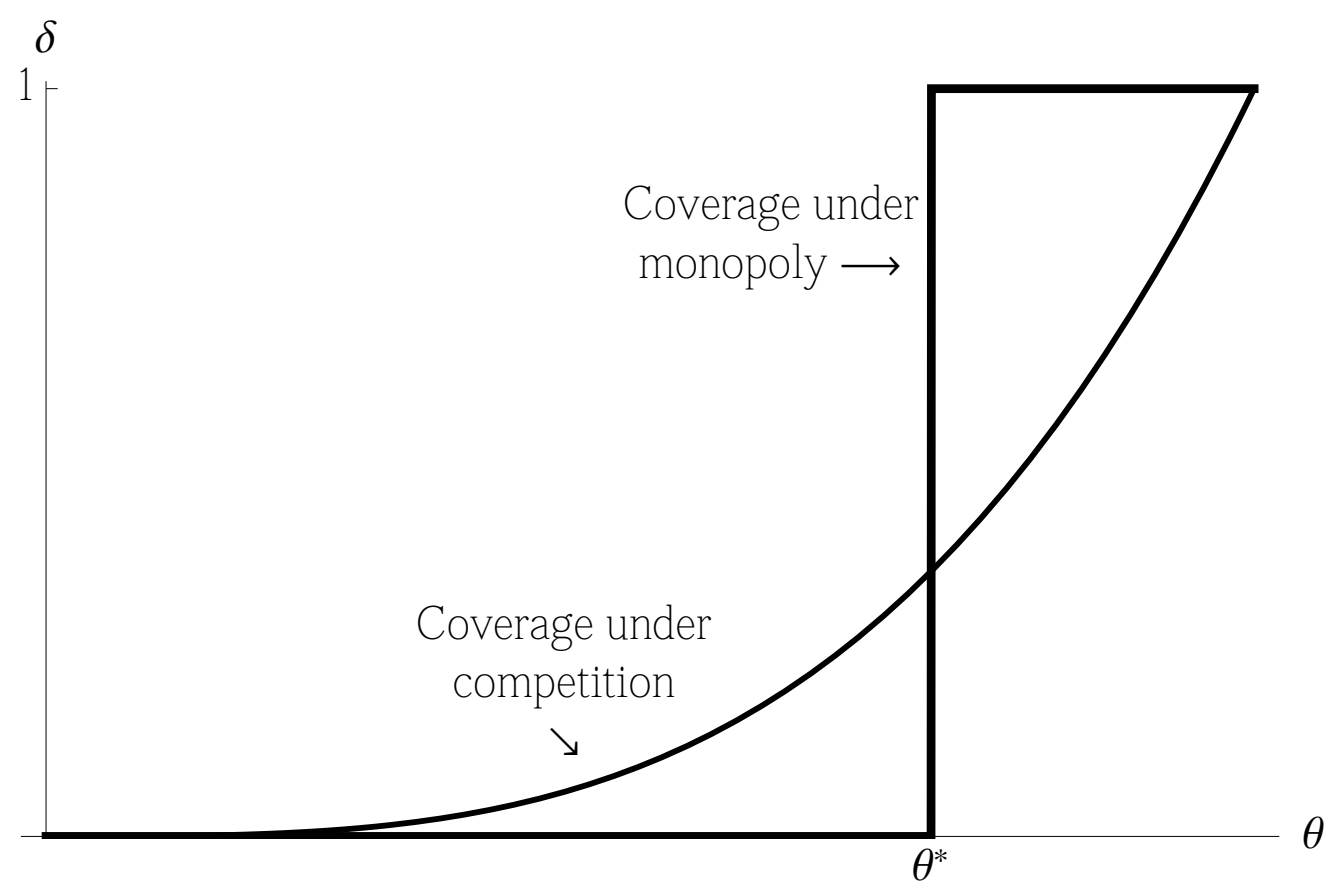

Figure 1: Relative coverage rates under competition and monopoly.

In Figure 2 the effect of a change in distribution is depicted: the left panel shows a positively skewed distribution, while the right panel shows a negatively skewed distribution. While the coverage function under competition is unchanged, the level of $\theta^{*}$ in the left panel is lower than in the right panel. This means that when the distribution of risks is positively skewed, the monopolist charges a lower premium then when it is negatively skewed. The gain for the monopolist of including more types in the pooling exceeds the profit loss due to the reduction in premium.

There is a clear trade-off between participation and coverage in the insurance markets under monopoly and competition. Monopoly, in fact, ensures full coverage to the individuals that buy insurance contracts, but this is obtained at the expenses of low risk types that do not participate. Under competition, on the other hand, each individual is provided with an insurance contract, but coverage is only partial. As a consequence, there is no clear analytical result when we compare monopoly and competition. We need to perform numerical simulations on the distribution of individuals and the next Section provides the results.

\section{Relative efficiency}

In this section we perform some numerical simulations using $\operatorname{Bet} a(a, b)$ distribution of risks which gives much flexibility on the shape of the distribution. Indeed, if $a>1$ and $b>1$ the distribution is unimodal; if $a<1$ and $b<1$ the distribution is U-shaped; if $a=1$ and $b=1$ the distribution is uniform. The degree of skewness increases with the difference $|a-b|$. The 

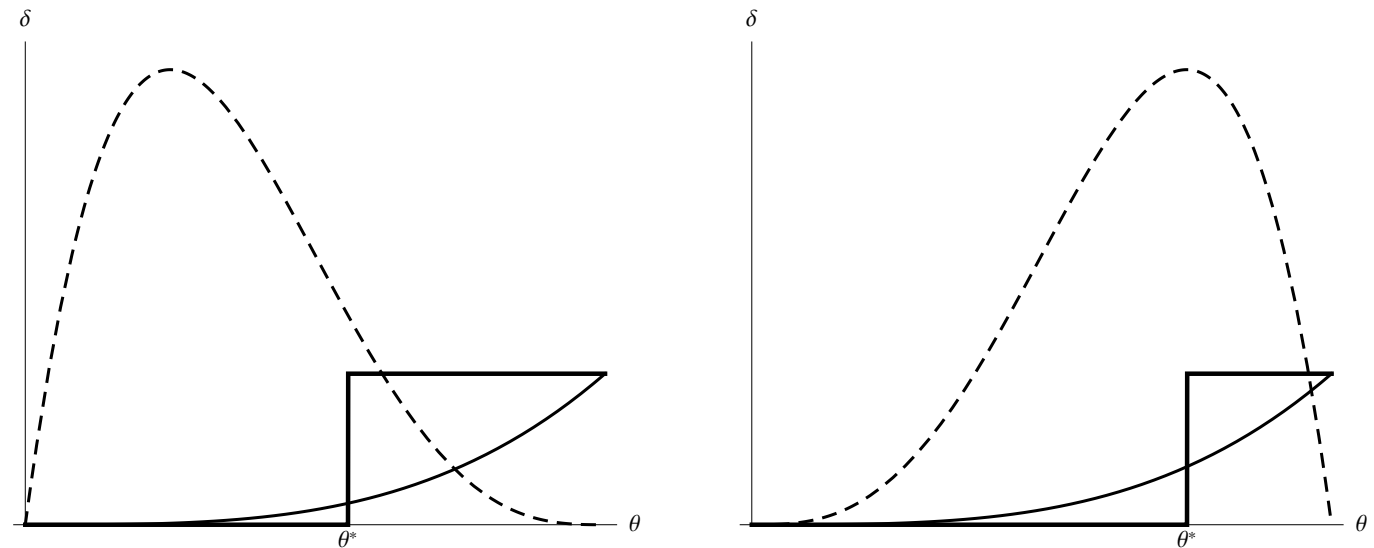

Figure 2: The effect of different distributions on coverage and participation under competition and monopoly.

\begin{tabular}{|c||c|c|c|c|c|}
\hline \multicolumn{1}{|c||}{} & \multicolumn{5}{c|}{$\mathrm{b}$} \\
\hline $\mathrm{a}$ & 1 & 2 & 3 & 5 & 10 \\
\hline \hline 1 & 20.00 & 11.11 & 7.87 & 5.29 & 3.46 \\
2 & 33.33 & 22.09 & 17.05 & 12.41 & 8.59 \\
3 & 42.86 & 31.35 & 25.54 & 19.64 & 14.22 \\
5 & 55.56 & 45.19 & 39.20 & 32.32 & 24.98 \\
10 & 71.43 & 64.24 & 59.49 & 53.20 & 44.98 \\
\hline
\end{tabular}

Table 1: Participation rate under monopoly for various distributions over risk Beta(a,b).

distribution is symmetric if $a=b$, positively skewed if $a<b$ and negatively skewed if $a>b$. We limit the analysis to the cases of $a, b \geq 1$ that ensure a non-decreasing hazard rate.

We have seen that the risk distribution affects the monopoly outcome by changing the critical level $\theta^{*}$, while it does not affect the competition equilibrium outcome.

The effect of changing the distribution on the equilibrium monopoly participation rate is illustrated in Table $1 .^{8}$ In this Table a Beta distribution $(a, b)$ is used to show that the participation rate increases with the concentration of the distribution (i.e., simultaneous increase in $a$ and $b$ ). Moreover, participation is higher with negatively skewed distributions (i.e., $a-b>0$ ). The intuition of the latter result is simple. Consider a small decrease in the skewness of the distribution such that the effect on $f\left(\theta^{*}\right)$ (a second order effect) is negligible. The first order effect of this change is an increase in $1-F\left(\theta^{*}\right)$. Then, at $\theta^{*}$ the monopoly is no longer in equilibrium since

$$
\theta^{*} h\left(\theta^{*}\right)<\frac{1+\alpha}{\alpha}
$$

and the firm can increase its profits by increasing the premium charged and, as a consequence,

\footnotetext{
${ }^{8}$ Table 1 and the following tables are built assuming $\alpha=1 / 3, \underline{\theta}=0$ and $\bar{\theta}=0.7$.
} 
the risk level of the critical type. At the new equilibrium

$$
\theta^{* *} h\left(\theta^{* *}\right)=\frac{1+\alpha}{\alpha}
$$

where $\theta^{* *}>\theta^{*}$, that implies $h\left(\theta^{* *}\right)<h\left(\theta^{*}\right)$. Given that the effect on $f\left(\theta^{*}\right)$ is negligible, it follows that the participation rate has increased; i.e., $\left[1-F\left(\theta^{* *}\right)\right]>\left[1-F\left(\theta^{*}\right)\right]$.

Following Rustichini et al. (1994) we measure efficiency in terms of total surplus generated in the market as a fraction of the first best (full information) surplus.

Table 2 shows the total surplus realized under competition and monopoly as a percentage of the total surplus under full information. Fixing the degree of risk aversion and the spread of risks we can compare competition and monopoly for different Beta distributions. The key result is that except for the uniform distribution $(a=b=1)$ and distributions for which the highest risk is the mode $(b=1)$, monopoly performs better than competition.

\begin{tabular}{|c||cc|cc|cc|cc|cc|}
\hline \multicolumn{1}{|c||}{} & \multicolumn{10}{c|}{$\mathrm{b}$} \\
\cline { 2 - 11 } & \multicolumn{2}{|c|}{1} & \multicolumn{2}{|c|}{2} & \multicolumn{2}{|c|}{3} & \multicolumn{2}{|c|}{5} & \multicolumn{2}{|c|}{10} \\
\hline \hline $\mathrm{a}$ & mon & comp & mon & comp & mon & comp & mon & comp & mon & comp \\
1 & 36.00 & 40.00 & 25.92 & 20.00 & 21.37 & 11.43 & 17.05 & 4.76 & 13.33 & 1.10 \\
2 & 45.57 & 50.00 & 35.51 & 28.57 & 30.43 & 17.86 & 25.20 & 8.33 & 20.23 & 2.20 \\
3 & 52.58 & 57.14 & 43.06 & 35.71 & 37.90 & 23.81 & 32.22 & 12.12 & 26.44 & 3.57 \\
5 & 62.21 & 66.67 & 54.01 & 46.67 & 49.15 & 33.94 & 43.34 & 19.58 & 36.73 & 6.86 \\
10 & 74.79 & 78.57 & 69.08 & 62.86 & 65.33 & 51.07 & 60.34 & 35.05 & 53.71 & 16.15 \\
\hline
\end{tabular}

Table 2: Surplus under monopoly and competition as a percentage of the First Best surplus for various distributions over risk Beta(a,b).

The difference in performance increases with the concentration and with the skewness (i.e., as $b-a$ increases). The level of the parameters may marginally affect the ranking and only in favor of monopoly. An increase in the risk aversion increases the surplus both under competition and monopoly. Under competition the coverage function $\delta^{c}(\theta)=(\theta / \bar{\theta})^{1 / \alpha}$ is increasing in $\alpha$; the intuition is that separation becomes easier and a lower difference in coverage suffices.

Under monopoly, in the equilibrium condition

$$
\theta^{*} h\left(\theta^{*}\right)=\frac{1+\alpha}{\alpha}
$$

the RHS is decreasing in $\alpha$ and so the equilibrium entails a lower $\theta^{*}$ and a higher participation rate. The intuition is that the willingness to pay increases with the risk aversion, and thus participation can increase with a smaller cut in premium.

It is possible to show with simulations that the ranking between monopoly and competition is not affected by any value of $\alpha \in(0,1) .{ }^{9}$ On the contrary, a reduction in the spread of

\footnotetext{
${ }^{9}$ It is worth recalling that $\alpha>1$ means that $\phi(\theta)>2 \theta$.
} 
risks $[\underline{\theta}, \bar{\theta}]$ may alter the ranking in favor of monopoly. Consider, for example, the uniform distribution case. In this case $h(\theta)=\frac{1}{\bar{\theta}-\theta}$ and the equilibrium condition becomes:

$$
\frac{\theta^{*}}{\bar{\theta}-\theta^{*}}=\frac{1+\alpha}{\alpha}
$$

So, if $\underline{\theta} \geq \frac{1+\alpha}{1+2 \alpha} \bar{\theta}$, then $\theta^{*} \equiv \underline{\theta}$ and all the individuals are provided with full insurance under monopoly - the first best solution; this result is not possible under competition.

Finally, it is worth emphasizing that both monopoly and competition do not implement neither constrained Pareto-optimal nor second-best allocations in terms of total surplus. On the one hand, implementable allocations under competition are reduced by the additional set of zero-profit constraints; on the other hand, monopolist firm maximizes profits rather than consumers or total surplus. A Pareto-dominant allocation with respect to the monopolist outcome is simply the allocation that increases the pool of types buying full insurance up to the point in which the premium paid is equal to the average cost; the allocation obviously increase total surplus, too. A Pareto-dominant allocation with respect to the competitive outcome is the one that pools the closest-to-the-highest risk types with the highest risk type in a full coverage contract up to the point in which the lowest risk in the pool is indifferent between the new pooling contract and the old competitive equilibrium contract intended for him, and leaves the same competitive equilibrium contracts to all the other (lower) risk types. ${ }^{10}$ This new allocation also increases the total surplus generated on the market.

\section{Extension to concave probability weighting functions}

The aim of this Section is to generalize the result of the previous Sections to the case of non linear weighting function. We will first identify the monopolist and competitive equilibria, and then we will analyze their relative efficiency.

The monopolist equilibrium result summarized in Proposition 1 is obtained with the linear probability weighting function $\phi(\theta)=(1+\alpha) \theta$. In the following Proposition we will generalize the result for any (weakly) concave probability weighting function such that $\phi(\theta)>\theta$, $\phi^{\prime}(\theta)>0$ and $\phi^{\prime \prime}(\theta) \leq 0$.

Proposition 3 For any concave probability weighting function $\phi(\theta)$, the monopoly market equilibrium outcome involves a unique $\theta^{*} \in[\underline{\theta}, \bar{\theta}]$ such that

$$
\phi\left(\theta^{*}\right)-\theta^{*}=\frac{\phi^{\prime}\left(\theta^{*}\right)}{h\left(\theta^{*}\right)}
$$

where $h(\theta)$ is the non-decreasing hazard function, and the insurance contracts are

$$
\begin{array}{ll}
\left\{\delta^{m}(\theta), \pi^{m}(\theta)\right\}=\{0,0\} & \forall \theta \in\left[\underline{\theta}, \theta^{*}\right] \\
\left\{\delta^{m}(\theta), \pi^{m}(\theta)\right\}=\left\{1, \phi\left(\theta^{*}\right)\right\} & \forall \theta \in\left[\theta^{*}, \bar{\theta}\right]
\end{array}
$$

\footnotetext{
${ }^{10}$ This allocation is always feasible; Riley (2001) shows how reducing the premium of the full coverage contract (and so attracting more risk types) is always profitable for any continuous distribution.
} 
Proof. See Appendix.

Proposition 3 shows therefore that the uniqueness of the contract offered by the monopolist is not specific to the particular (linear) functional form taken by the probability weighting function used in this paper, but it is a general result under Yaari's dual theory of choice under risk. Attar et al. (2011, Lemma 1) show that the same result is obtained in the context of nonexclusive contract and it is due to the linearity of the preferences with respect to wealth. ${ }^{11}$

Shifting to the analysis of competition, the following Proposition generalizes the competitive equilibrium solution of Proposition 2 for any (weakly) concave probability weighting function.

Proposition 4 For any concave probability weighting function $\phi(\theta)$, the competitive equilibrium outcome involves the set of contracts

$$
\left\{\delta^{c}(\theta), \pi^{c}(\theta)\right\}=\left\{\delta^{c}(\theta), \theta \delta^{c}(\theta)\right\}
$$

where

$$
\delta^{c}(\theta)=\exp -\int_{\theta}^{\bar{\theta}} \frac{1}{\phi(s)-s} d s
$$

\section{Proof. See Appendix}

The result that monopoly generally provides higher social surplus than competition is obtained assuming that individuals have preferences which can be described using the Yaari dual theory of choice under risk, and assuming a linear probability weighting function. This choice is useful because it makes surplus comparison more straightforward but it also provides a benchmark when we want to extend the conclusions to a more general setting with a probability transformation function $\phi(\theta)$ such that $\theta<\phi(\theta)<1$ and $\phi^{\prime}(\theta)>0 \forall \theta$. We can in fact show that whenever the probability weighting function is concave the outcome worsen for competition with respect to monopoly.

\subsection{Linear equivalent of $\phi($.$) that preserves the monopoly equilibrium \theta^{\star}$}

Assume that the probability transformation function is twice continuously differentiable with $\theta<\phi(\theta)<1, \phi^{\prime}(\theta)>0$ and $\phi^{\prime \prime}(\theta)<0 \forall \theta \in[\underline{\theta}, \bar{\theta}]$.

Define $\theta^{*}$ the lowest type buying the full-coverage insurance contract offered by a monopolist at the price $\pi^{*}=\phi\left(\theta^{*}\right)$. Now define

$$
\psi(\theta)=\left(1+\alpha^{*}\right) \theta \text { where } \alpha^{*} \text { is such that } \theta^{*}=\frac{1+\alpha^{*}}{\alpha^{*} h\left(\theta^{*}\right)} .
$$

In other words, we are assuming that $\psi(\theta)$ is a linear probability transformation function such that the monopolist optimal contract is the same as with $\phi(\theta)$. So the same pool of types $\theta \in\left[\theta^{*}, \bar{\theta}\right]$ buys the full insurance contract offered by the monopolist with both $\phi(\theta)$ and $\psi(\theta)$ probability transformation functions. The two functions are represented in Figure 3.

\footnotetext{
${ }^{11}$ See also Samuelson (1984) and Myerson (1985).
} 


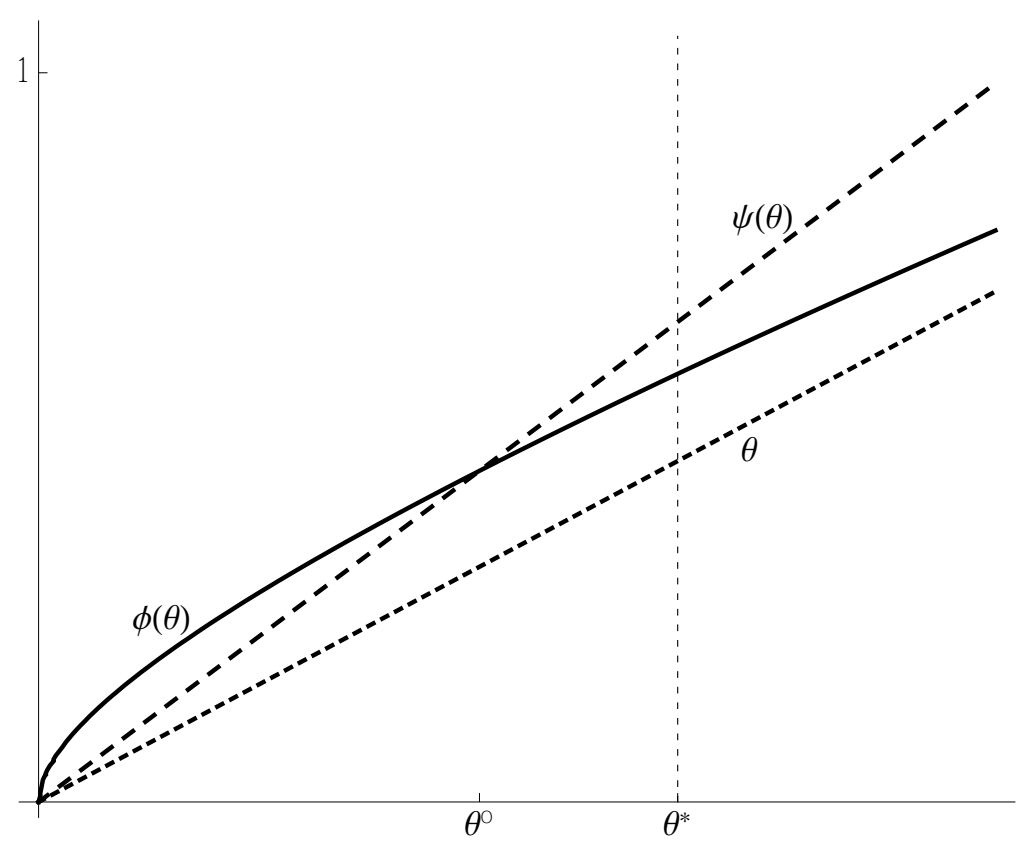

Figure 3: A concave probability transformation function $\phi(\theta)$ and the linear function $\psi(\theta)$ which preserves the willingness to pay for $\theta^{*}$.

It is to be noted that, in order to keep $\theta^{*}$ constant, it is necessary that $\psi\left(\theta^{*}\right)>\phi\left(\theta^{*}\right)$. This is easily seen from the monopolist first-order condition associated to probability transformation functions

$$
\begin{aligned}
\psi\left(\theta^{*}\right)-\theta^{*} & =\frac{\psi^{\prime}\left(\theta^{*}\right)}{h\left(\theta^{*}\right)} \\
\phi\left(\theta^{*}\right)-\theta^{*} & =\frac{\phi^{\prime}\left(\theta^{*}\right)}{h\left(\theta^{*}\right)}
\end{aligned}
$$

Taking difference,

$$
\psi\left(\theta^{*}\right)-\phi\left(\theta^{*}\right)=\frac{\psi^{\prime}\left(\theta^{*}\right)-\phi^{\prime}\left(\theta^{*}\right)}{h\left(\theta^{*}\right)}>0
$$

since by concavity $\phi^{\prime}(\theta)<\psi^{\prime}(\theta)$ for all $\theta$ to the right of the intersection point $\psi\left(\theta^{\circ}\right)=\phi\left(\theta^{\circ}\right) .{ }^{12}$

Therefore, since $\psi(\theta)>\phi(\theta) \forall \theta \in\left(\theta^{\circ}, \bar{\theta}\right]$, we have that

$$
\int_{\theta}^{\bar{\theta}} \frac{1}{\phi(s)-s} d s>\int_{\theta}^{\bar{\theta}} \frac{1}{\psi(s)-s} d s \quad \forall \theta \in\left[\theta^{i}, \bar{\theta}\right]
$$

where $\theta^{i}$, if exists, is such that

\footnotetext{
${ }^{12}$ It is always the case that $\theta^{\circ}<\theta^{*}$ because otherwise the monopolist would found profitable to increase the premium and have a smaller pool of risk types buying insurance.
} 


$$
\int_{\theta^{i}}^{\bar{\theta}} \frac{1}{\phi(s)-s} d s=\int_{\theta^{i}}^{\bar{\theta}} \frac{1}{\psi(s)-s} d s
$$

This means that

$$
\delta^{c}(\phi(.), \theta)<\delta^{c}(\psi(.), \theta) \quad \forall \theta \in\left(\theta^{i}, \bar{\theta}\right]
$$

and so the surplus generated in the competitive equilibrium with the linear probability transformation function $\psi($.$) is generally much larger than the one generated with the corre-$ sponding concave probability transformation function $\phi(.) .{ }^{13}$ Therefore, any concave probability transformation $\phi(\theta)$ that preserves the monopoly outcome, reduces the amount of insurance bought in the competitive equilibrium, and thus generates a lower surplus with competition. In Figure 4 we represent the monopoly and competitive equilibrium coverage functions obtained with the probability transformation functions $\phi(\theta)$ and $\psi(\theta)$ used in Figure 3.

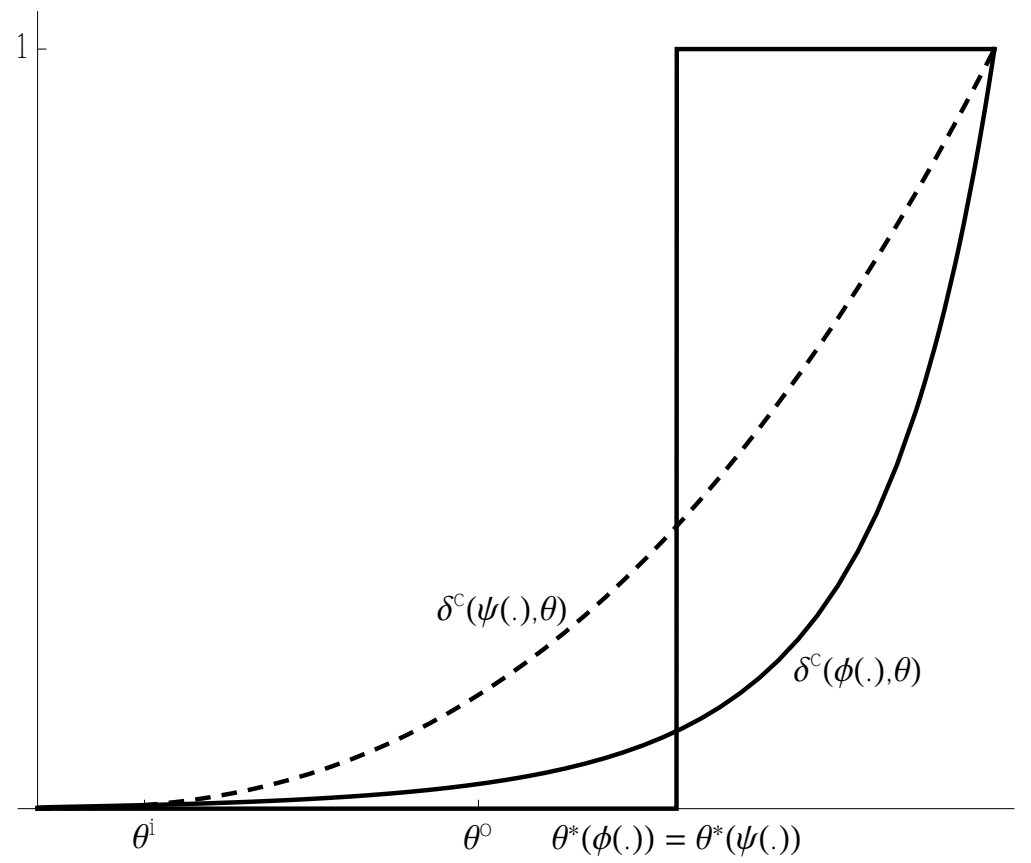

Figure 4: The monopolist and competitive equilibria obtained with the probability transformation functions $\phi(\theta)$ and $\psi(\theta)$ used in Figure 3.

The results that competition can hurt efficiency is thus reinforced with a concave probability weighting function. It is worth noting that the concave probability weighting function is consistent with a marginal willingness to pay for insurance that is decreasing with the amount of insurance bought. This is also a central feature of the standard expected utility model.

\footnotetext{
${ }^{13}$ Even though the competitive equilibrium does not depend on the distribution of types, $\theta^{*}$ does, and therefore the linear transformation $\psi($.$) depends on the distribution. For all the distributions used in these$ paper we found that $\theta^{i}$ either does not exist or is very small and that the competitive equilibrium surplus with $\psi($.$) is much larger than the one with \phi($.$) .$
} 


\section{Conclusions}

Using the benchmark model of Rothschild and Stiglitz (1976), we contrast the competitive equilibrium outcome with the monopoly equilibrium outcome à la Stiglitz (1977) and we compare their relative efficiency. The main change is that we adopt the dual theory of risk so that the comparison comes out neatly when dealing with a continuum of types. The dual theory has the property that utility is linear in income, and risk aversion is expressed entirely by a transformation of probabilities in which bad outcomes are given relatively higher weights and good outcomes are given relatively lower weights.

Our main finding is that competition is bad and that the monopoly outcome in general is more efficient than the competitive outcome (according to our expected efficiency criterion defined as the fraction of the total surplus that is realized by the market). The reason why monopoly performs better than competition is that the monopolist can exploit its market power to relax the incentive constraints. In this paper, following the general view that competition fully separates risk types in equilibrium, we identify the competitive outcome as the Pareto-dominant full-separating zero-profits set of contracts, which is the (candidate) equilibrium according to several approaches including Rothschild and Stiglitz (1976), Reactive and Walrasian equilibrium concepts, and the models developed by Dubey and Geanakoplos (2002) and Hellwig (1987). However, an available alternative is the equilibrium concept developed by Wilson (1977), Miyazaki (1977), and Spence (1978) (henceforth WMS). ${ }^{14}$ The WMS equilibrium concept allows for cross-subsidization under competition and Crocker and Snow (1985) show it is always second-best efficient. Therefore the WMS equilibrium always performs better than the monopolist equilibrium, which is not second-best efficient. This results confirms the benefits of cross-subsidization in the presence of adverse selection, and our contribution provides also an additional insight in the debate about competitive equilibria. When competition allows for cross-subsidization the equilibrium outcome is second-best but, if the competitive pressure is such that no cross-subsidization can occur, then the outcome is even worse than the one achieved by a monopoly.

This is one of many examples of the interplay between market imperfections. The economy, in effect has to trade off between two different imperfections: imperfections of information or imperfections of competition, with no particular reason that these imperfections will be balanced optimally.

We expect our result about the inefficiency of competition in insurance markets with adverse selection to carry over on other markets with adverse selection like the capital market or the job market.

There is a final remark about the use of the dual theory of risk. With this specification there is no income effect on the demand of insurance. In contrast, the expected utility approach will raise the demand for insurance in the monopoly market relative to the competitive market if the absolute risk aversion is decreasing. This is because monopoly price is higher than

\footnotetext{
${ }^{14}$ See Netzer and Scheuer (2012) and Mimra and Wambach (2011) for game-theoretic foundation for the WMS equilibrium.
} 
competitive price which reduces income and thus raises the marginal willingness to pay for insurance. It is then expected that moving to the expect utility will further increase the amount of insurance in the monopoly market relative to the competitive market, thereby reinforcing our conclusion about the inefficiency of competition.

\section{Appendix}

Proof of Propositions 1 and 3. We will develop the proof using the generic probability weighting function $\phi(\theta)$ and then substitute eventually for the specific weighting function used. So,

$$
V(\theta ; \delta, \pi)=\omega-\pi-\phi(\theta)(1-\delta)
$$

Because the unique binding IR constraint is for the lowest type:

$$
V(\underline{\theta} ; \delta(\underline{\theta}), \pi(\underline{\theta}))=V(\underline{\theta} ; 0,0) .
$$

The set of incentive constraints implies that

$$
\theta \in \arg \max _{\hat{\theta} \in[\underline{\theta}, \bar{\theta}]} V(\theta ; \delta(\hat{\theta}), \pi(\hat{\theta})) \quad \forall \theta, \hat{\theta} \in[\underline{\theta}, \bar{\theta}] \text { with } \hat{\theta} \neq \theta
$$

the first order condition for the type $\theta$ is:

$$
\begin{aligned}
\frac{\partial V(\theta ; \delta(\hat{\theta}), \pi(\hat{\theta}))}{\partial \hat{\theta}} & =\frac{\partial}{\partial \hat{\theta}}[\omega-\pi(\hat{\theta})-\phi(\theta)(1-\delta(\hat{\theta}))] \\
& =\phi(\theta) \delta^{\prime}(\hat{\theta})-\pi^{\prime}(\hat{\theta})=0
\end{aligned}
$$

which evaluated at $\hat{\theta}=\theta$ gives the local incentive compatibility conditions (LIC)

$$
\phi(\theta) \delta^{\prime}(\theta)-\pi^{\prime}(\theta)=0 \quad \forall \theta \in[\underline{\theta}, \bar{\theta}]
$$

Moreover, the necessary LIC is also sufficient condition when the utility function satisfies the increasing differences property,

$$
\frac{\partial^{2} V(\theta ; \delta(\hat{\theta}), \pi(\hat{\theta}))}{\partial \hat{\theta} \partial \theta}=\phi^{\prime}(\theta) \delta^{\prime}(\hat{\theta}) \geq 0
$$

which requires the coverage to be monotonically increasing $\delta^{\prime}(\theta) \geq 0$, whenever $\phi^{\prime}(\theta)>0 .{ }^{15}$

Define the value function of the maximization problem evaluated at the truth-telling equilibrium:

$$
U(\theta)=V(\theta ; \delta(\theta), \pi(\theta))=\omega-\phi(\theta)(1-\delta(\theta))-\pi(\theta)
$$

differentiating with respect to $\theta$

$$
\begin{aligned}
U^{\prime}(\theta) & =-\phi^{\prime}(\theta)(1-\delta(\theta))+\phi(\theta) \delta^{\prime}(\theta)-\pi^{\prime}(\theta) \\
& =-\phi^{\prime}(\theta)(1-\delta(\theta))<0
\end{aligned}
$$

\footnotetext{
${ }^{15}$ Throughout the paper we assume that $\phi^{\prime}(\theta)>0$ so that the single crossing property holds for any $\theta \in[\underline{\theta}, \bar{\theta}]$.
} 
where the second equality follows from (11).

Using these results we can rewrite the maximization programme of the monopolist as follows:

$$
\max _{\pi(\theta), \delta(\theta)} \int_{\underline{\theta}}^{\bar{\theta}}[\pi(\theta)-\delta(\theta) \theta] d F(\theta)
$$

subject to

$$
\begin{aligned}
\delta^{\prime}(\theta) & \geq 0 \\
\phi(\theta) \delta^{\prime}(\hat{\theta})-\pi^{\prime}(\hat{\theta}) & =0 \\
V(\underline{\theta} ; \delta(\underline{\theta}) ; \pi(\underline{\theta})) & \geq V(\underline{\theta} ; 0,0)
\end{aligned}
$$

Ignoring for the moment the monotonicity constraint that will be checked later, we can rewrite the objective function after substituting the constraints in it. From the definition of the value function (12)

$$
\pi(\theta)=\omega-\phi(\theta)(1-\delta(\theta))-U(\theta)
$$

and by equation (13)

$$
U(\theta)=U(\underline{\theta})+\int_{\underline{\theta}}^{\theta}-\phi^{\prime}(s)(1-\delta(s)) d s
$$

By using the binding constraint for the low type,

$$
\begin{aligned}
U(\theta) & =\omega-\phi(\underline{\theta})-\phi(\theta)+\phi(\underline{\theta})+\int_{\underline{\theta}}^{\theta} \phi^{\prime}(s) \delta(s) d s \\
& =\omega-\phi(\theta)+\int_{\underline{\theta}}^{\theta} \phi^{\prime}(s) \delta(s) d s
\end{aligned}
$$

Substituting this expression into (14):

$$
\begin{aligned}
\pi(\theta) & =\omega-\phi(\theta)(1-\delta(\theta))-\omega+\phi(\theta)-\int_{\underline{\theta}}^{\theta} \phi^{\prime}(s) \delta(s) d s \\
& =\phi(\theta) \delta(\theta)-\int_{\underline{\theta}}^{\theta} \phi^{\prime}(s) \delta(s) d s
\end{aligned}
$$

This expression for the insurance premium captures both the incentive and participation constraints. Plugging this premium in the objective function we get the reduced problem:

$$
\max _{\delta(\theta)} \int_{\underline{\theta}}^{\bar{\theta}}\left[\phi(\theta) \delta(\theta)-\delta(\theta) \theta-\int_{\underline{\theta}}^{\theta} \phi^{\prime}(s) \delta(s) d s\right] d F(\theta)
$$

The second term is the aggregate informational rent which integrating by parts is given by

$$
\int_{\underline{\theta}}^{\bar{\theta}} \int_{\underline{\theta}}^{\theta} \phi^{\prime}(s) \delta(s) d s d F(\theta)=\left|\int_{\underline{\theta}}^{\theta} \phi^{\prime}(s) \delta(s) d s F(\theta)\right|_{\underline{\theta}}^{\bar{\theta}}-\int_{\underline{\theta}}^{\bar{\theta}} \phi^{\prime}(\theta) \delta(\theta) F(\theta) d \theta
$$


with

$$
\begin{aligned}
\int_{\underline{\theta}}^{\underline{\theta}} \phi^{\prime}(s) \delta(s) d s & =0 \\
F(\underline{\theta}) & =0 \\
F(\bar{\theta}) & =1
\end{aligned}
$$

Hence:

$$
\begin{aligned}
\int_{\underline{\theta}}^{\bar{\theta}} \int_{\underline{\theta}}^{\theta} \phi^{\prime}(s) \delta(s) d s d F(\theta) & =\int_{\underline{\theta}}^{\bar{\theta}} \phi^{\prime}(\theta) \delta(\theta) d \theta-\int_{\underline{\theta}}^{\bar{\theta}} \phi^{\prime}(\theta) \delta(\theta) F(\theta) d \theta \\
& =\int_{\underline{\theta}}^{\bar{\theta}} \frac{1-F(\theta)}{f(\theta)} \phi^{\prime}(\theta) \delta(\theta) d F(\theta)
\end{aligned}
$$

Plugging the solution for the informational rent into the objective function

$$
\max _{\delta(\theta) \in[0,1]} \int_{\underline{\theta}}^{\bar{\theta}}\left[\phi(\theta) \delta(\theta)-\delta(\theta) \theta-\frac{1-F(\theta)}{f(\theta)} \phi^{\prime}(\theta) \delta(\theta)\right] d F(\theta)
$$

Let $h(\theta)=\frac{f(\theta)}{1-F(\theta)}$ be the hazard rate, then the monopoly programme is

$$
\max _{\delta(\theta) \in[0,1]} \int_{\underline{\theta}}^{\bar{\theta}} \delta(\theta)\left[\phi(\theta)-\theta-\frac{\phi^{\prime}(\theta)}{h(\theta)}\right] d F(\theta)
$$

Because the objective is maximized when the argument of the integral is maximized $\forall \theta \in[\underline{\theta}, \bar{\theta}]$ the result of Proposition 3 is obtained.

Furthermore, by substituting $\phi(\theta)$ with $(1+\alpha) \theta$, the monopolist problem (17) becomes

$$
\max _{\delta(\theta) \in[0,1]} \int_{\underline{\theta}}^{\bar{\theta}} \delta(\theta)\left[\alpha \theta-\frac{1-\alpha}{h(\theta)}\right] d F(\theta)
$$

and the result of Proposition 1 is proven.

It remains to check the monotonicity constraint. To be verified, it requires that

$$
\frac{\partial^{2}}{\partial \delta(\theta) \partial \theta}\left[(\phi(\theta)-\theta) \delta(\theta)-\frac{\phi^{\prime}(\theta)}{h(\theta)} \delta(\theta)\right] \geq 0
$$

With a linear probability transformation function, it simplifies to

$$
\frac{h^{\prime}(\theta)}{h^{2}(\theta)} \geq-\frac{\alpha}{1+\alpha}
$$

and a sufficient condition is that the hazard rate is non-decreasing in the interval $[\underline{\theta}, \bar{\theta}]$, which completes the proof.

With a general probability transformation function, in addition to the monotonicity of the hazard rate, the following sufficient condition ensures the monotonicity of the coverage function:

$$
\phi^{\prime}(\theta)>1+\phi^{\prime \prime}(\theta) h(\theta)
$$


Proof of Propositions 2 and 4. From the zero profit conditions (7) it follows that the participation constraints (2) are not binding and can be disregarded.

Following the incentive compatibility approach of Mailath (1987) the set (3) can be rewritten as the maximization programme of agents

$$
\theta \in \arg \max _{\hat{\theta} \in[\underline{\theta}, \bar{\theta}]} V(\theta ; \delta(\hat{\theta}), \pi(\hat{\theta})) \quad \forall \theta, \hat{\theta} \in[\underline{\theta}, \bar{\theta}]
$$

By the zero profit condition (7), every agent with risk $\theta$ is facing actuarially fair premium: $\pi(\theta)=\delta(\theta) \theta$. This can be incorporated in the local incentive compatibility condition for the type $\theta$ that has been derived in equation (11) in the proof of Propositions 1-3, which now becomes: ${ }^{16}$

$$
\phi(\theta) \delta^{\prime}(\theta)-\delta(\theta)-\theta \delta^{\prime}(\theta)=0 \quad \forall \theta \in[\underline{\theta}, \bar{\theta}]
$$

Hence the equilibrium coverage rate function $\delta(\theta)$ is the solution to the following differential equation,

$$
\frac{\delta^{\prime}(\theta)}{\delta(\theta)}=\frac{1}{\phi(\theta)-\theta} \quad \forall \theta \in[\underline{\theta}, \bar{\theta}]
$$

Let $r(\theta)=\frac{1}{\phi(\theta)-\theta}$. The differential equation can be solved as follows. Integrating the LHS yields

$$
\int \frac{\delta^{\prime}(\theta)}{\delta(\theta)} d \theta=\log \delta(\theta)+c_{1}
$$

while integrating the RHS yields

$$
R(\theta)=\int r(\theta) d \theta
$$

Then, taking exponential on both sides gives

$$
\delta(\theta)=k e^{R(\theta)}
$$

where $k$ is the constant of integration. Using the no distortion at the top condition, we have the terminal condition, $\delta(\bar{\theta})=1$, so that

$$
\delta(\bar{\theta})=k e^{R(\bar{\theta})}=1 \Longrightarrow k=e^{-R(\bar{\theta})}
$$

So, the unique solution is

$$
\delta^{c}(\theta)=e^{R(\theta)-R(\bar{\theta})}
$$

where $R(\theta)-R(\bar{\theta})=-\int_{\theta}^{\bar{\theta}} r(s) d s$, which completes the proof of Proposition 4. To prove Proposition 1 requires to use the linear probability transformation function $\phi(\theta)=(1+\alpha) \theta$

\footnotetext{
${ }^{16}$ As before, the necessary LIC is also sufficient condition when coverage function is monotonically increasing.
} 
and so:

$$
\begin{aligned}
r(\theta) & =\frac{1}{\phi(\theta)-\theta}=\frac{1}{\alpha \theta} \\
R(\theta) & =\int \frac{1}{\phi(\theta)-\theta} d \theta=\log \theta^{\frac{1}{\alpha}} \\
e^{R(\theta)} & =\theta^{\frac{1}{\alpha}} \\
e^{R(\bar{\theta})} & =\bar{\theta}^{\frac{1}{\alpha}}
\end{aligned}
$$

Therefore

$$
\delta^{c}(\theta)=e^{R(\theta)-R(\bar{\theta})}=(\theta / \bar{\theta})^{\frac{1}{\alpha}} \in[0,1] \quad \forall \theta \in[\underline{\theta}, \bar{\theta}]
$$

By the zero profit condition the equilibrium premium function is

$$
\pi^{c}(\theta)=\delta^{c}(\theta) \theta=\left(\frac{\theta^{1+\alpha}}{\bar{\theta}}\right)^{\frac{1}{\alpha}} \quad \forall \theta \in[\underline{\theta}, \bar{\theta}]
$$

Since the coverage function is increasing in $\theta$, the second order condition is respected, which completes the proof of Proposition $2 .{ }^{17}$

\section{References}

Akerlof, George A., "The Market for "Lemons": Quality Uncertainty and the Market Mechanism," Quarterly Journal of Economics, August 1970, 84 (3), 488-500.

Attar, Andrea, Thomas Mariotti, and François Salanié, "Nonexclusive Competition in the Market for Lemons," Econometrica, 2011, 79 (6), 1869-1918.

Bisin, Alberto and Piero Gottardi, "Efficient Competitive Equilibria with Adverse Selection," Journal of Political Economy, June 2006, 114 (3), 485-516.

Chiappori, Pierre-André, "The Welfare Effects of Predictive Medicine," in Pierre-André Chiappori and Christian Gollier, eds., Competitive Failures in Insurance Markets: Theory and Policy Implications, CESifo Seminar Series, Cambridge, MA: The MIT Press, July 2006.

Crocker, Keith J. and Arthur Snow, "The efficiency of competitive equilibria in insurance markets with asymmetric information," Journal of Public Economics, March 1985, 26 (2), $207-219$.

Dahlby, Bev G., "Monopoly Versus Competition in an Insurance Market with Adverse Selection," Journal of Risk \& Insurance, June 1987, 54 (2), 325-331.

De Donder, Philippe and Jean Hindriks, "Adverse selection, moral hazard and propitious selection," Journal of Risk and Uncertainty, 2009, 38 (1), 73-86.

${ }^{17}$ In fact, $\delta^{c \prime}(\theta)=\frac{1}{\alpha}\left(\frac{\theta^{1-\alpha}}{\bar{\theta}}\right)^{\frac{1}{\alpha}} \geq 0 \quad \forall \theta \in[\underline{\theta}, \bar{\theta}]$. 
de Meza, David and David C Webb, "Advantageous Selection in Insurance Markets," RAND Journal of Economics, Summer 2001, 32 (2), 249-62.

Dubey, Pradeep and John Geanakoplos, "Competitive Pooling: Rothschild-Stiglitz Reconsidered," Quarterly Journal of Economics, November 2002, 117 (4), 1529-1570.

Engers, Maxim and Luis Fernandez, "Market Equilibrium with Hidden Knowledge and Self-Selection," Econometrica, March 1987, 55 (2), 425-439.

Hellwig, Martin, "Some recent developments in the theory of competition in markets with adverse selection," European Economic Review, February - March 1987, 31 (1-2), 319-325.

Hindriks, Jean and Philippe De Donder, "The politics of redistributive social insurance," Journal of Public Economics, December 2003, 87 (12), 2639-2660.

Jaffe, Dwight and Joseph E. Stiglitz, "Credit Rationing," in Benjamin M. Friedman and Frank H. Hahn, eds., Handbook of Monetary Economics, 2, Handbooks in Economics series, Amsterdam, NL: North Holland, November 1990, pp. 837-888.

Jullien, Bruno, Bernard Salanié, and François Salanié, "Screening risk-averse agents under moral hazard: single-crossing and the CARA case," Economic Theory, 2007, 30 (1), $151-169$.

Mailath, George J., "Incentive Compatibility in Signaling Games with a Continuum of Types," Econometrica, November 1987, 55 (6), 1349-1365.

Mimra, Wanda and Achim Wambach, "A Game-Theoretic Foundation for the Wilson Equilibrium in Competitive Insurance Markets with Adverse Selection," Working Paper Series 3412, CESifo, Munich: Germany April 2011.

Miyazaki, Hajime, "The rat race and internal labor markets," Bell Journal of Economics, Autumn 1977, 8 (2), 394-418.

Myerson, Roger B., "Analysis of Two Bargaining Problems with Incomplete Information," in Alvin E. Roth, ed., Game Theoretic Models of Bargaining, Cambridge: UK: Cambridge University Press, 1985, pp. 115-147.

Netzer, Nick and Florian Scheuer, "A Game Theoretic Foundation of Competitive Equilibria with Adverse Selection," Working Paper 18471, National Bureau of Economic Research October 2012.

Prescott, Edward C. and Robert M. Townsend, "Pareto Optima and Competitive Equilibria with Adverse Selection and Moral Hazard," Econometrica, 1984, 52 (1), pp. $21-46$.

Riley, John G., "Informational Equilibrium," Econometrica, March 1979, 47 (2), 331-360. 
_, "Silver Signals: Twenty-Five Years of Screening and Signaling," Journal of Economic Literature, June 2001, 39 (2), 432-478.

Rothschild, Michael and Joseph E. Stiglitz, "Equilibrium in Competitive Insurance Markets: An Essay on the Economics of Imperfect Information," Quarterly Journal of Economics, November 1976, 90 (4), 629-649.

Rustichini, Aldo, Mark A. Satterthwaite, and Steven R. Williams, "Convergence to Efficiency in a Simple Market with Incomplete Information," Econometrica, September 1994, 62 (5), 1041-1063.

Samuelson, William, "Bargaining under Asymmetric Information," Econometrica, 1984, 52 (4), pp. 995-1005.

Sandroni, Alvaro and Francesco Squintani, "Overconfidence, Insurance, and Paternalism," American Economic Review, 2007, 97 (5), 1994-2004.

_ and _ , "Overconfidence and asymmetric information: The case of insurance," Journal of Economic Behavior $\&$ Organization, 2012, forthcoming,--.

Spence, Michael, "Product differentiation and performance in insurance markets," Journal of Public Economics, 12 1978, 10 (3), 427-447.

Spinnewijn, Johannes, "Insurance and Perceptions: How to Screen Optimists and Pessimists," The Economic Journal, 2013, 123 (569), 606-633.

Stiglitz, Joseph E., "Information and Economic Analysis," in Michael J. Parkin and Avelino R. Nobay, eds., Current Economic Problems, New York, NJ: Cambridge University Press, 1975, pp. 27-52.

- , "Monopoly, Non-linear Pricing and the Imperfect Information: The Insurance Market," Review of Economic Studies, October 1977, 44 (138), 407-430.

von Siemens, Ferdinand A., "Social Preferences, Sorting, and Competition," The Scandinavian Journal of Economics, 2012, 114 (3), 780-807.

Wilson, Charles, "A model of insurance markets with incomplete information," Journal of Economic Theory, December 1977, 16 (2), 167-207.

Yaari, Menahem E., "The Dual Theory of Choice under Risk," Econometrica, January 1987, 55 (1), 95-115. 